\title{
A study of Self Organized Criticality in ITG mode driven gyrokinetic turbulence
}

\author{
M. Mavridis, ${ }^{1}$ H. Isliker, ${ }^{1}$ L. Vlahos, ${ }^{1}$ T. Görler, ${ }^{2}$ F. Jenko, ${ }^{2}$ and D. Told ${ }^{2}$ \\ 1) Section of Astrophysics, Astronomy and Mechanics, Department of Physics, Aristotle University of Thessaloniki, \\ GR-54124 Thessaloniki, Greece \\ 2) Max Planck Institute for Plasma Physics, Boltzmannstr. 2, 85748 Garching, \\ Germany
}

(Dated: 30 September 2014)

An investigation on the characteristics of self organized criticality (Soc) in ITG mode driven turbulence is made, with the use of various statistical tools (histograms, power spectra, Hurst exponents estimated with the rescaled range analysis and the structure function method). For this purpose, local non-linear gyrokinetic simulations of the Cyclone Base Case scenario are performed with the GENE software package. Although most authors concentrate on global simulations, which seem to be a better choice for such an investigation, we use local simulations in an attempt to study the locally underlying mechanisms of Soc. We also study the structural properties of radially extended structures, with several tools (fractal dimension estimate, cluster analysis, and two dimensional autocorrelation function), in order to explore whether they can be characterized as avalanches. We find that, for large enough driving temperature gradients, the local simulations exhibit most of the features of Soc, with the exception of the probability distribution of observables, which show a tail, yet they are not of power-law form. The radial structures have the same radial extent at all temperature gradients examined, radial motion (transport) though appears only at large temperature gradients, in which case the radial structures can be interpreted as avalanches.

Keywords: Self-organized criticality — gyrokinetics — plasma turbulence — confined plasma

\section{INTRODUCTION}

The mechanisms responsible for anomalous transport in magnetically confined plasmas are a subject of main interest in fusion research. The characteristic scale of the transport when considering the diffusive model proposed e.g., by Kadomtsev predicts a gyro-Bohm scaling ${ }^{1}$, which is of the order of the correlation length of turbulence. Experimental results show that the scale can though extend to Bohm scaling, where the dominant transport scale length is of the order of the machine size. One of the mechanisms suggested ${ }^{2}$ to explain the apparent paradox is based on the ideas of self-organized criticality ${ }^{3}$ (Soc).

Self-organized criticality is a possible state of complex, spatially extended systems that are systematically driven and that have mechanisms to develop local instabilities and to relax them. A main characteristic of Soc systems are long-range spatial and temporal correlations ${ }^{2-5}$, indicating scale invariance and self-similarity. They are manifested in the so called avalanches i.e., ballistically propagating structures in various observables. When SOC is present in dynamical systems, $1 / f$ noise is generated due to the propagation of local perturbations over all length and time scales which usually cause sub critical transport $^{6}$. Observables of a system in a Soc state exhibit power law form of their probability distribution function.

The possible presence of self organized criticality in magnetically confined plasmas has been studied in many ways. The creation and simulation of models that have self organized criticality characteristics and the comparison of the findings with measurements from experimental devices is an approach extensively used. One- dimensional transport models ${ }^{7}$, transport models derived from sandpile models ${ }^{8}$, cellular automata models as running sandpile models ${ }^{4}$, diffusive sandpile cellular automata models ${ }^{9}$, cellular automata models derived from a diffusion equation ${ }^{10}$, as well as many other models have been proposed. There are also studies that search for the characteristics of self organized criticality in experimental data. Electrostatic, density or temperature fluctuations are measured by Langmuir probes usually at the plasma edge of Tokamaks or Stellarators. The analysis of measurements from the SOL of DIII-D ${ }^{11-13}$, TCABR ${ }^{14}$, Tore Supra and Castor ${ }^{15}$, TEXTOR ${ }^{16}, \mathrm{KT}-5 \mathrm{D}^{17}$ are some of the examples in this line of work. Summarizing some of the experimental findings concerning self-organized criticality we mention that the power spectrum of various observables, e.g. density or electrostatic potential fluctuations, exhibits a $1 / f$ behavior, and the radial propagation of avalanches is observed. Also, long-range time correlations (or self-similarity) have been detected.

In this work, we are going to search for the characteristics of Soc in data from numerical simulations with a gyrokinetic code, which is the main tool of many studies. We are using the software package GENE (Gyrokinetic Electromagnetic Numerical Experiment) ${ }^{19-22}$ for performing non-linear ITG mode driven, gyrokinetic simulations in local magnetic flux tube geometry. Although local simulations do not seem to be the best candidate for the study of self organized criticality characteristics, since the latter is a global phenomenon, it is proposed in a work by McMillan et al. (Ref. 18) that a flux tube like 1D model can be used to study the characteristics of bursts and that the underlying mechanisms of avalanches are local processes. So far, only the appearance of avalanches 
has been reported in gyrokinetic simulations by many authors in various kinds of simulations, i.e. avalanches are detected in local ${ }^{23}$ and global ${ }^{24}$ gyrokinetic simulations. There have also been studies using gyrokinetic simulations concerning the effect of rotation on avalanches ${ }^{25}$, and mainly on how avalanches influence transport ${ }^{26-28}$. There has not yet been made though a statistical study of the time series of observables for an examination of all the characteristics of self organized criticality, besides the appearance of avalanches. Our purpose here is to perform such a statistical examination of gyrokinetic simulations using various statistical tools. We also explore the dependence of Soc characteristics on the different physical parameters and the set up used in gyrokinetic simulations.

The rest of this paper is organized as follows. In Sec. II the numerical set up of our gyrokinetic simulations is given, in Sec. III we show the results of the analysis of the time series of the radially averaged heat flux, applying various statistical tools, and searching for the characteristics of self organized criticality. In Sec. IV we preform a two dimensional analysis of the heat flux in the radius - time $(x-t)$ plane, focusing our interest on structural properties of avalanches, in Sec. V a brief discussion and comments on the results are given, and we summarize our findings in Sec. VI. A detailed description of the theoretical framework of the statistical tools used is given in Appendix A.

\section{BASIC PARAMETERS AND SET UP}

Our goal is to find the characteristics of a Soc state in gyrokinetic simulations of ITG mode driven turbulence, and as already stated, we consider the case of local nonlinear simulations. Since we focus on ITG turbulence we will use a variation of the basic paradigm used in the literature, the well known Cyclone Base $\mathrm{Case}^{29}$ (from now on referred to as $\mathrm{CBC}$ ). We use a magnetic equilibrium with circular ${ }^{30}$ concentric flux-surfaces and with inverse aspect ratio $a / R=0.36$. This of course differs from the CBC scenario, which uses s-alpha geometry but this selection was made because we need a common basis for planned future global simulations which can only be done in circular geometry with the GENE software package. Local simulations are taking place in a magnetic flux tube and the center of this magnetic flux tube is the point where the parameter values are assumed. Also, electrons are considered to be adiabatic in order to have only ion driven modes of turbulence. The local safety factor is selected to have the usual value for the CBC, $q_{0}=1.4$ with shear $s=0.796$ at $r=0.5 a$ and the local aspect ratio is set to $\epsilon_{t}=0.18$. The value for the plasma beta $\beta$ is chosen for the case of the electrostatic limit so $\beta=0$. The density gradient is set to the value $\omega_{n}=2.22$ and the temperature gradient varies in the range $\omega_{T}=3.5-6.9$ in different simulations, with all the other parameters kept the same. We also assume that the plasma is collision-less.

The perpendicular to the magnetic field simulation box size is chosen to be $\left(L_{x}, L_{y}\right)=(125.628,125.664)$ in units of the ion gyroradius $\rho_{s}$ and $128 \times 48 \times 24$ grid points are used in the radial $(x)$, binormal $(y)$, and parallel direction $(z)$, respectively, complemented by $32 \times 8$ grid points in $\left(v_{\|}, \mu\right)$ space, where $v_{\|}$is the parallel velocity and $\mu$ the magnetic moment. Finally, the box size in the $v_{\|}$direction is $L_{v}=3.0$ in units of the thermal velocity, and the upper limit in $\mu$ direction is $L_{\mu}=9.0$ in units of the equilibrium temperature of the species and the inverse reference magnetic field. The minimum $k_{y}$ mode is typically set in the simulations to the value $k_{y \text { min }}=0.05$. Finally for time integration, a Runge - Kutta scheme of the fourth order is used.

For the parametric study, we have chosen the minimum value of the temperature gradient $\omega_{T}=3.5$ just above the critical temperature gradient threshold of linear local ITG simulations $\left(\omega_{T}=3.1\right)$ for the most unstable mode which is close to $k_{y}=0.2 \sim 0.3$, and we then use different values of $\omega_{T}$ up to the usual $\mathrm{CBC}$ value $\omega_{T}=6.9$. The parameters of the set up are summarized in Table I.

TABLE I: Summary of the parameters used in the non-linear gyrokinetic simulations preformed with the software package GENE.

\begin{tabular}{cr}
\hline \hline Basic parameters & Numerical value \\
\hline Temperature gradient $R / L_{T}$ & $3.5-6.9$ \\
\hline Density gradient $R / L_{n}$ & 2.22 \\
\hline Magnetic shear $\hat{s}$ & 0.796 \\
\hline Safety factor $q_{0}$ & 1.4 \\
\hline Inverse aspect ratio $\epsilon$ & 0.36 \\
\hline Inverse aspect ratio (local) $\epsilon_{t}$ & 0.18 \\
\hline \hline
\end{tabular}

\section{RESULTS FROM THE TIME SERIES ANALYSIS}

\section{A. Analysis}

We analyze the time series of the radially averaged heat flux. Because of the varying time step used by GENE, we have interpolated the data using cubic splines and created time series that are equally spaced in time. We exclude the initial linear growth phase of the time series and select a part in it, where we can say that there is saturation and stationarity ( an example is presented in Fig. 1). The latter is important since it is a prerequisite for some of the analysis tools applied. The number of data points used in the analysis is 65536 , a power of 2 , useful for Fourier transforms. 


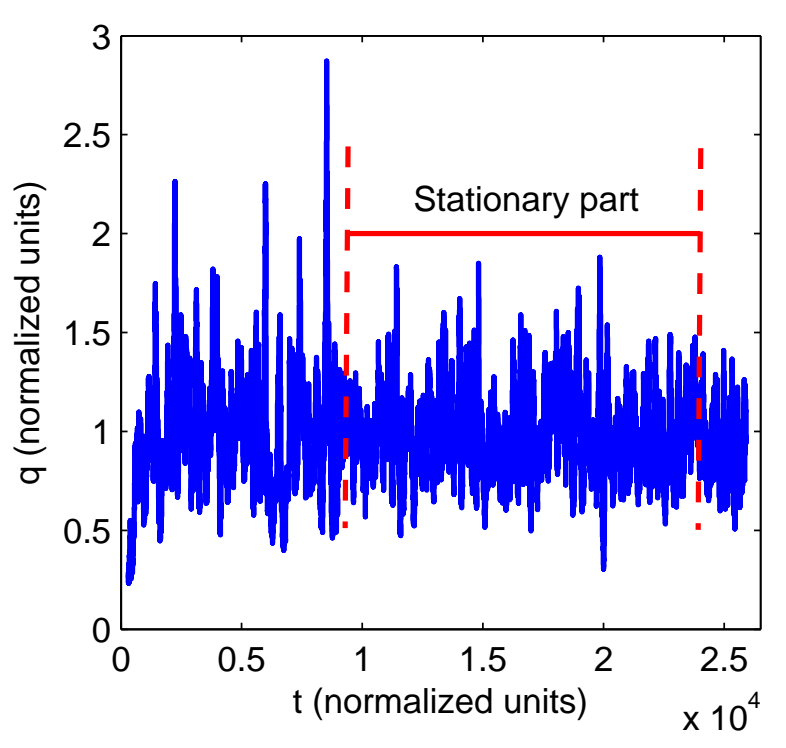

FIG. 1: (Color online) A sample time series of the radially averaged heat flux $q$ for temperature gradient $R / L_{T}=5.0$. Red dashed lines mark the stationary part of the time series selected to be analyzed.

\section{B. Histograms, skewness and kurtosis}

We determine the histogram from each time series (normalized to unity). Some sample histograms for selected cases of simulations are presented in Fig. 2. Each histogram is not an exact Gaussian but has an asymmetry, as can be expected in turbulence. The histograms are shifted and spread in width as the temperature gradient increases. The latter holds also in the case of the histograms not shown. The extent of the tail is too small for a fit to be applied. The shapes of the histograms are not compatible with Soc systems where the probability distributions of observables have a power law form.

For a quantification of the asymmetry in the distributions we measure the skewness and the excess kurtosis, as explained in Appendix A, using Eq. A9, A11, respectively. The variation of the skewness and the excess kurtosis with the temperature gradients is shown in Fig. 3 and as can been seen, it is not a smooth function of the temperature gradient.

All histograms are positively skewed and they also possess positive kurtosis. The positive skew indicates the existence of relatively large events (events larger than the mean value of the time series), even for the smallest temperature gradient values. We can see that the value of the skewness does not change significantly with the increasing temperature gradient, which suggests that the appearance of large events does not change significantly with the increase of the gradient. The kurtosis seems to increase slightly with the increase of the temperature gradient. This increase indicates that there is an increase in the peakedness and tail which could be related to the

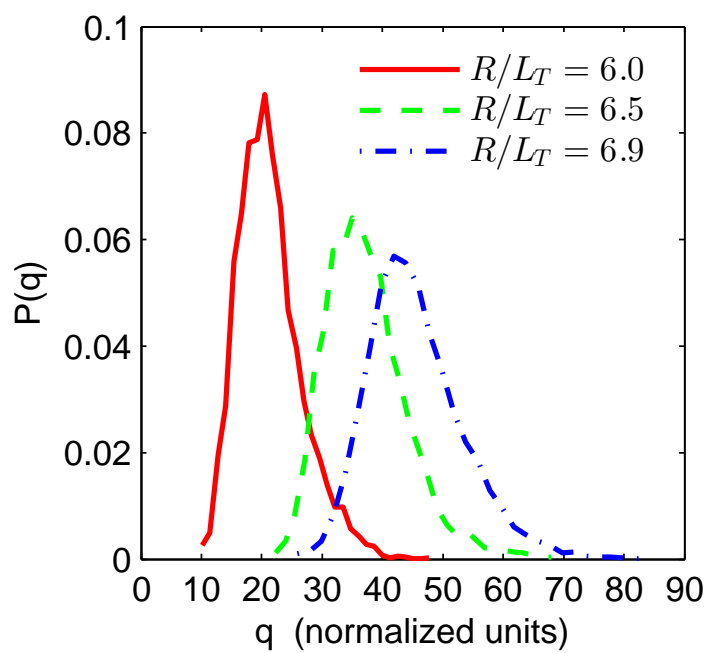

FIG. 2: (Color online) Probability distribution functions $P(q)$ of sample histograms of the normalized heat flux $q$ for the gradient values, $R / L_{T}=6.0$ (red) solid line, $R / L_{T}=6.5$ (green) dashed line, $R / L_{T}=6.9$

(blue) dashed-dotted line.

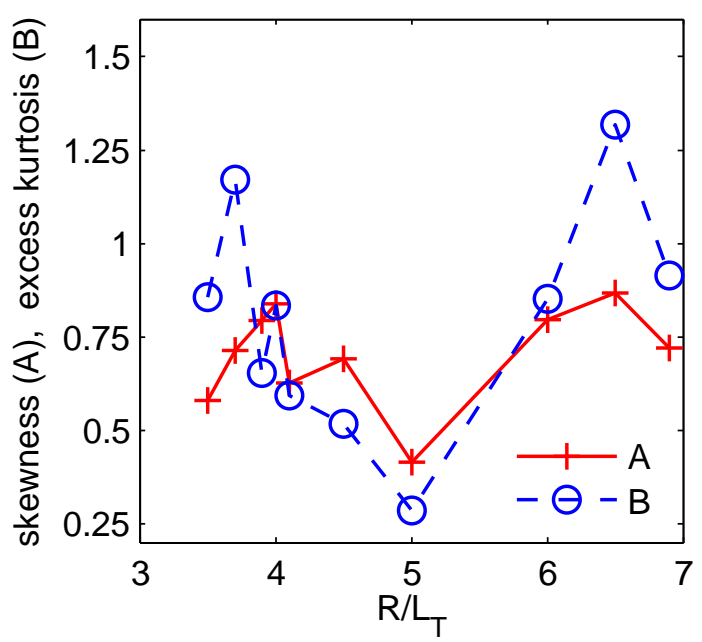

FIG. 3: (Color online) Variation of skewness (red) solid line with crosses (A) and excess kurtosis (blue) dashed line with circles (B) with the temperature gradient.

increase of the scales of structures created in more developed turbulence.

\section{Power spectrum}

A useful tool in the study of Soc systems is the power spectrum of the time series of observables. The power spectrum of such systems, can be divided into several distinct frequency regions where it follows power laws, $P(w) \propto w^{-\alpha}$, with varying exponents. Each region is related to a different scale of events taking place. At 
low frequencies a power law exponent equal to zero is expected corresponding to decorrelated scales. The region of intermediate frequencies with exponent equal to unity has been related to the overlapping of avalanches, and finally the region of large frequencies with an exponent much larger than 2, corresponds to small scale events ${ }^{32}$. This behavior has been reported in theoretical works e.g., in Refs. 7 and 9, and it has also been seen in the power spectrum of experimental data e.g., in Refs. 16, 17.

We determine the power spectrum of the radially averaged heat flux. In Fig. 4, the power spectrum from three simulations with different temperature gradients is presented. The multiple power law feature, characteristic of Soc systems, is observed, and appears also in the power spectrum of the simulations not shown here. As the temperature gradient increases the power spectrum is expanding to higher frequencies, which means that even smaller scales appear in the system. There is an increase of the highest frequency of almost a decade, when $R / L_{T}$ increases from $R / L_{T}=3.5$ to $R / L_{T}=6.9$.

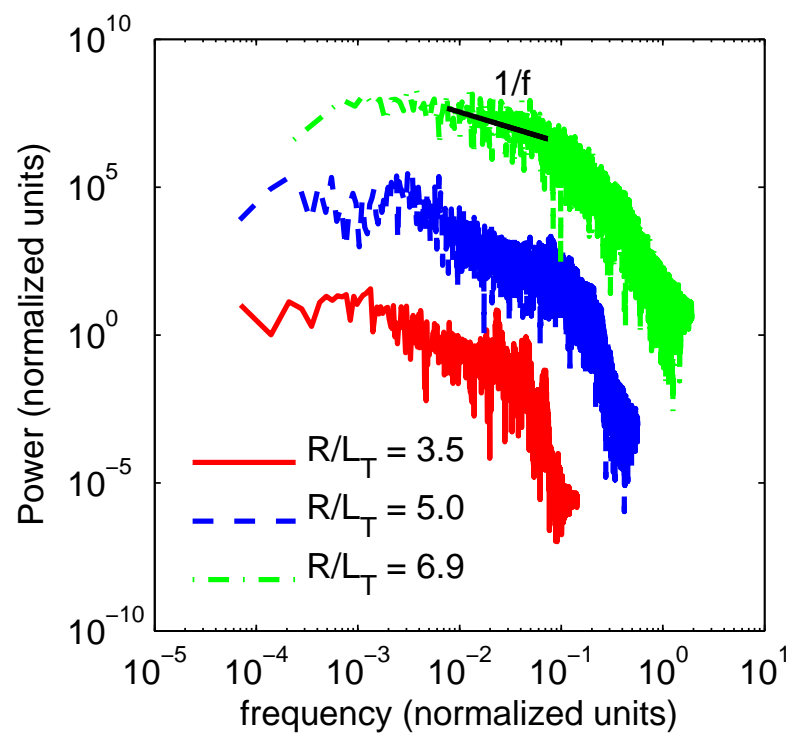

FIG. 4: (Color online) Power spectrum of the heat flux from simulations for the cases with temperature gradients: (a) solid line (red) $R / L_{T}=3.5$, (b) dashed line (blue) $R / L_{T}=5.0$, (c) dashed-dotted line (green) $R / L_{T}=6.9$.

We identify in the power spectra the three distinct regions. The region at low frequencies shows exponents very close to or almost zero. The region at large frequencies exhibits exponent values starting from high values at low temperature gradients $(\alpha \sim 10)$ and as the temperature gradient increases the value of the exponent decreases to smaller values $(\alpha \sim 5-6)$.

The exponent of the power law at the intermediate frequencies, is of main interest. The variation of the exponent in this region with the temperature gradient is shown in Fig. 5. It can be seen that the exponent is varying between the values 1 and 3 . As the temperature gradient increases the characteristic for Soc systems $1 / f$ dependency, appears and the extent of the intermediate frequency region increases. This can be explained by examining the number of $k_{y}$ modes in Fourier space for various observables but mainly the heat flux. We find that almost all $k_{y}$ modes included in the set up of our simulations are non linearly interacting with a significant amplitude when the temperature gradient $R / L_{T} \geq 5.0$, while only a small number of modes interacts non linearly with significant amplitude at lower gradients. Also, by examining the contribution of each $k_{y}$ mode to the heat flux, we can see that all of them contribute when $R / L_{T} \geq 5.0$, while for lower gradients only the linearly most unstable modes are important for the heat flux.

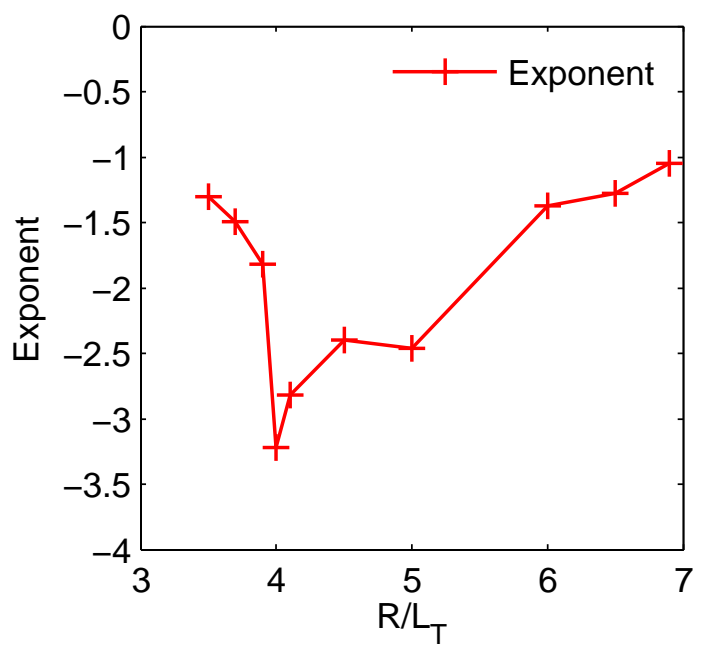

FIG. 5: (Color online) Variation of the power-law exponent of the power spectrum in the intermediate range frequencies (red) solid line.

\section{Hurst exponent from the rescaled range analysis}

A calculation of the Hurst exponent using the rescaled range analysis ${ }^{33}$ (see App. A) method gives three different scaling regions, as expected from the shape of the power spectrum. An example of the rescaled range analysis is given in Fig. 6. There is a region where the exponent is close to unity (region A), an intermediate region which is of main interest (region B) and finally a region where the values of the Hurst exponent are approaching 0 (region $\mathrm{C}$ ).

The variation of the Hurst exponent for each region with the temperature gradient is presented in Fig. 7. In region $\mathrm{A}$, all simulations have a Hurst exponent close to unity. This region corresponds to highly correlated small scales. Region $\mathrm{C}$ corresponds to large decorrelated scales. We can see that the value of the Hurst exponent is quite high in region $\mathrm{C}$, we would expect it to be closer to zero 


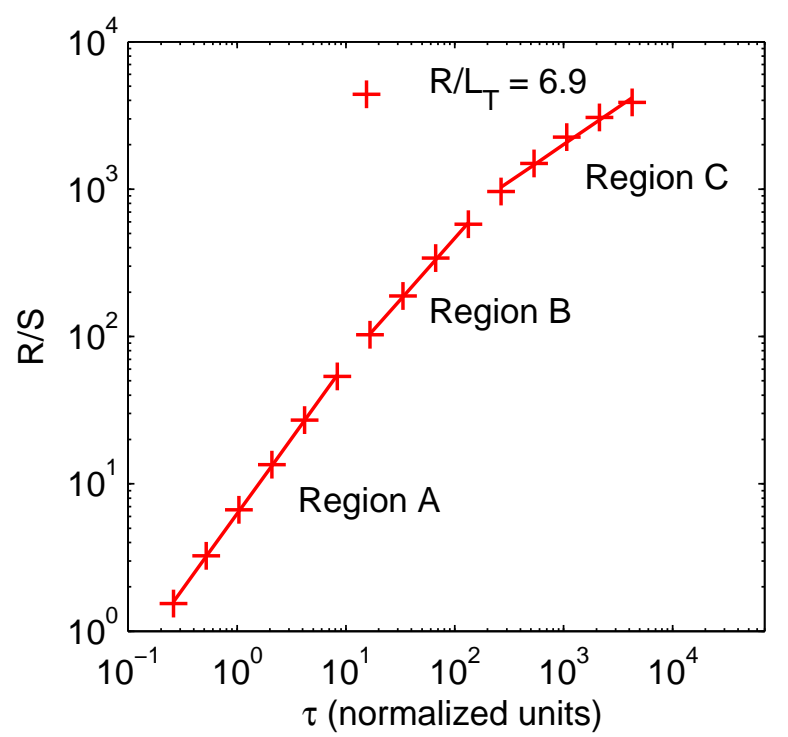

FIG. 6: (Color online) Rescaled range analysis as a function of the scale $\tau$. Three different regions with power-law scaling are discernible.

and only at the larger values of the temperature gradient, it starts at least diminishing towards the anticipated values.

The region we are mainly interested in is the middle region, the so called mesoscale region. The term mesoscale and its importance is discussed e.g. in Ref. 12. In short, the mesoscale is the intermediate scaling range, and it is of interest since at smaller scales the Hurst exponent is basically one, due to the strong, yet trivial, correlations at small scales in deterministic systems, while at larger scales the Hurst exponent basically equals zero, since the scales have been reached where the data become uncorrelated. The mesoscale thus is the scaling range that reveals non-trivial, long range correlations.

At low value temperature gradients, where turbulence is not fully developed, we can see that the Hurst exponent in the mesoscale region is close to unity. This means that the system is highly correlated and the corresponding fractal dimension D is close to unity according to Eq. A3. With increasing temperature gradient, which, as already stated, increases the number of the linearly destabilized modes, and also the number of nonlinearly interacting $k_{y}$ modes with significant amplitude that contribute to the turbulence seen in the heat flux, we can identify a clear deviation from unity and also an extension of the scaling region. The Hurst exponent is $H=0.85-0.83$ for gradients $R / L_{T} \geq 6.0$ something that shows the existence of long range correlations and persistence in the time series. We can also see that as the temperature gradient increases, the Hurst exponent slightly decreases. These values correspond to a fractal dimension $D=1.15-1.17$, according to Eq. A3.

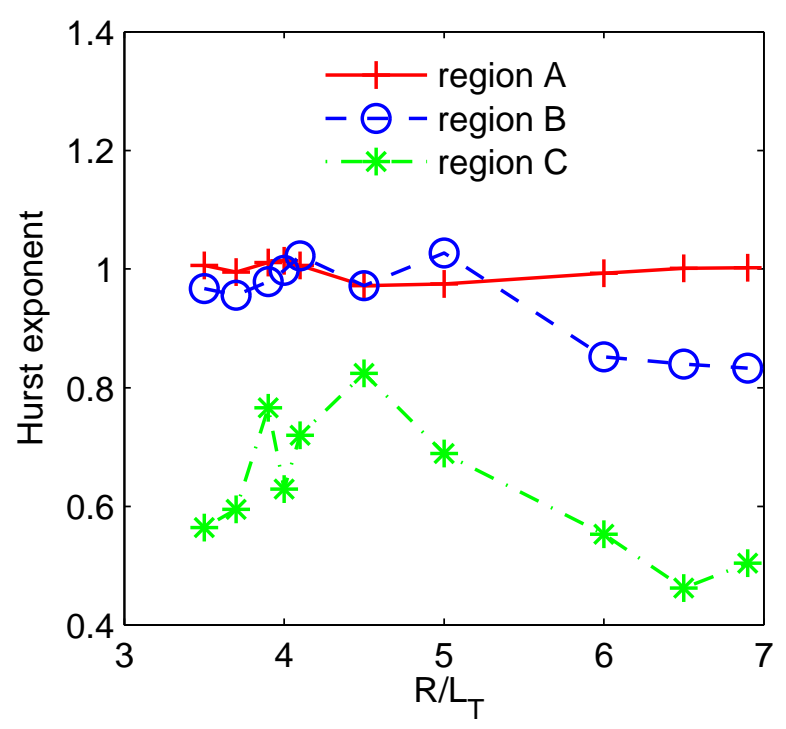

FIG. 7: (Color online) Variation of the Hurst exponent from the rescaled range analysis in the different scaling regions (see Fig. 6).

\section{E. Generalized Hurst exponents}

The Structure function ${ }^{34} S_{q}$, (see App. A) is another tool used to investigate long range correlations through generalized Hurst exponents. Structure functions offer many advantages ${ }^{12}$ compared to the rescaled range analysis method, among which are the inference of stationarity of a time series and of multifractality. We calculate the structure functions with the value of the parameter $q$ varying in the range $q=0.5-7$ and using first the raw (original), time series data. We then repeat the procedure for the cumulative data. From the structure functions of the raw data we can identify the region where the slope of $\log \left(S_{q}\right)$ with $\log (\tau)$, where $\tau$ is the time lag, is equal to zero for all $q$, which shows the scale range where the raw time series is stationary. We then calculate the structure functions of the integrated data and estimate the Hurst exponent with linear fits in the log-log plots at almost the same $r$ egion where stationarity was detected in the raw data, for the different values of the $q$ parameter. An example is given in Fig. 8.

The analysis with the structure functions gives similar results as the rescaled range analysis above. Three distinct scaling regions appear, in agreement with the $R / S$ method. The first small $\tau$ region corresponds again to highly correlated small scales. The intermediate mesoscale region, which is of main interest, concerns self-similar structures. The large $\tau$ region, corresponds to the large decorrelated scales.

In all simulations, the first region exhibits a generalized Hurst exponent equal to unity, for all the values of the $q$ parameter. The largest $\tau$ region has values of the Hurst exponent lower than or almost equal to $H \leq 0.5$. 

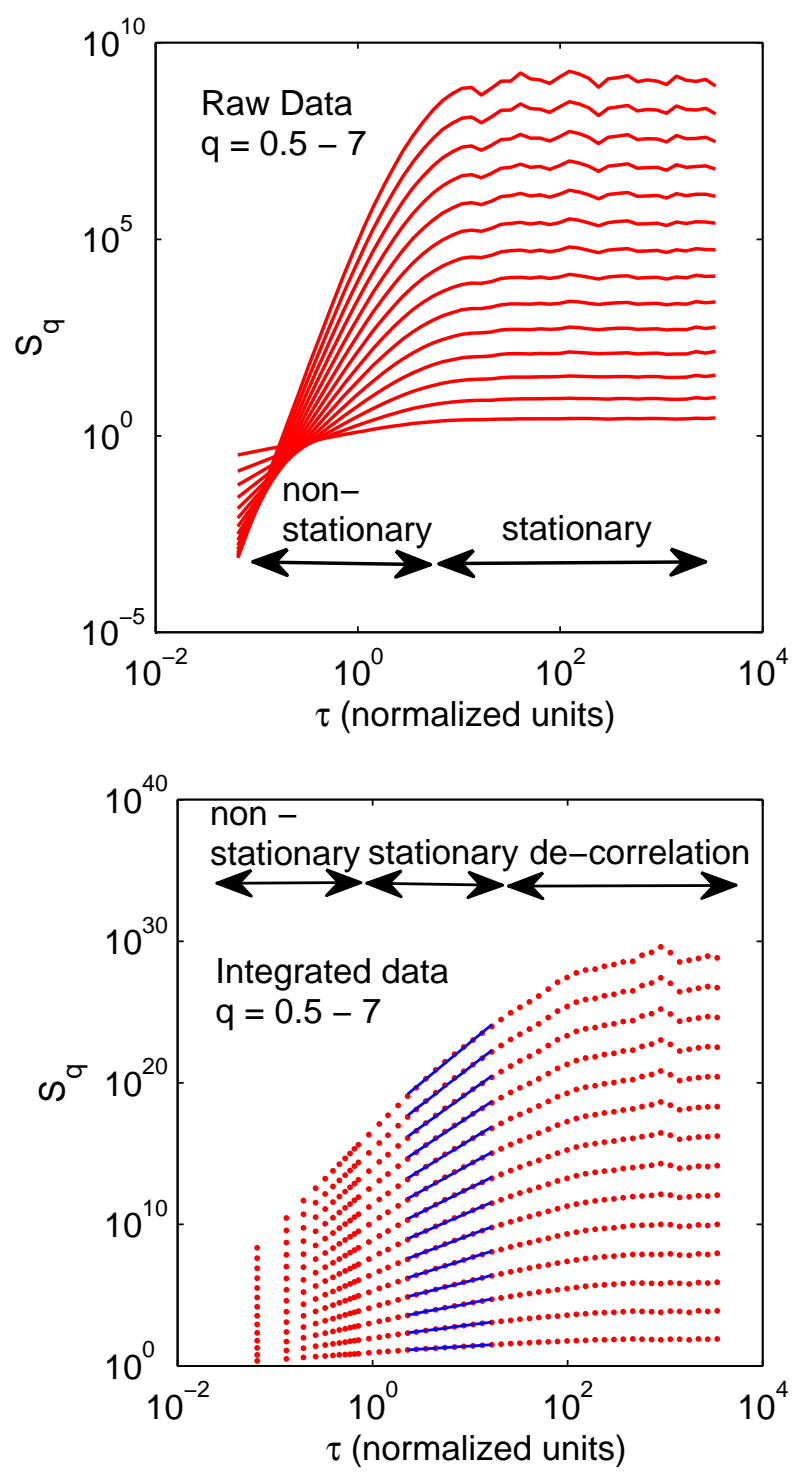

FIG. 8: (Color online) (top) Structure function of the original data against the time lag $\tau$. (bottom) Structure function of the cumulative data (red), dots, against the time lag $\tau$, with linear fits (blue), solid lines, in the mesoscale region. The lowest sequence of dots are for $q=0$, and $q$ increases in steps of 0.5, up to the upper most sequence of points which is for $q=7.0$

For the mesoscale region, we present the variation of the generalized Hurst exponent with the temperature gradient for the value of $q=2$ in Fig. 9. Estimates of the Hurst exponent with the structure function with parameter $q=2$ should theoretically give the same results as the estimates of the Hurst exponent calculated by the rescaled range analysis. At low temperature gradients we have values of the generalized Hurst exponent close to the value $H_{q} \sim 0.9$ and as the temperature gradient increases and turbulence gets fully developed, the value lowers to $H_{q} \sim 0.85$. These values are indeed very close to the estimates yielded by the $R / S$ method (see Fig. 7), and show persistence of the time series, and the existence of long range correlations.

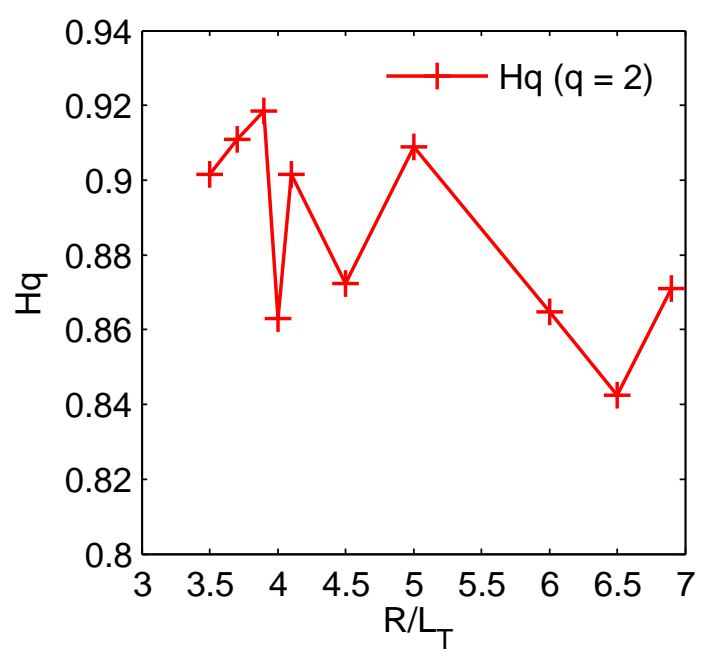

FIG. 9: (Color online) Variation of the generalized Hurst exponent for the value of $q=2$ with the temperature gradient $R / L_{T}$

The structure function method, as already stated, is also a useful tool for the detection of multifractality. to this end, we consider the product $q H_{q}=\zeta(q)$ as a function of $q$. If $\zeta(q)$ is a linear function of $q$ then we infer monofractality, and otherwise multifractality. An example is given in Fig. 10, for the case with $R / L_{T}=6.9$. We can see that $\zeta(q)$ is a linear function, and the same functional form is observed in all simulated cases, there is thus no multifractal scaling in all cases.

\section{RESULTS FROM THE ANALYSIS IN THE RADIUS TIME PLANE}

A main characteristic of systems in Soc state are avalanches. For their detection and the study of their statistics we perform some analysis in the radius time plane. The tools used are calculation of the fractal dimension of the heat flux patterns, analysis of the number and extent of radial structures, and calculation of the two dimensional autocorrelation function.

\section{A. Fractal dimension of avalanching structures}

We consider the heat flux as function of radius $(x)$ and time $(t)$, as computed by GENE. The analysis was made for the stationary parts of the heat flux data as determined for the time series in Sec. III, and we use simulations with varying temperature gradient $R / L_{T}=3.5-6.5$ in steps of 0.5 , except for the value $R / L_{T}=5.5$ where 


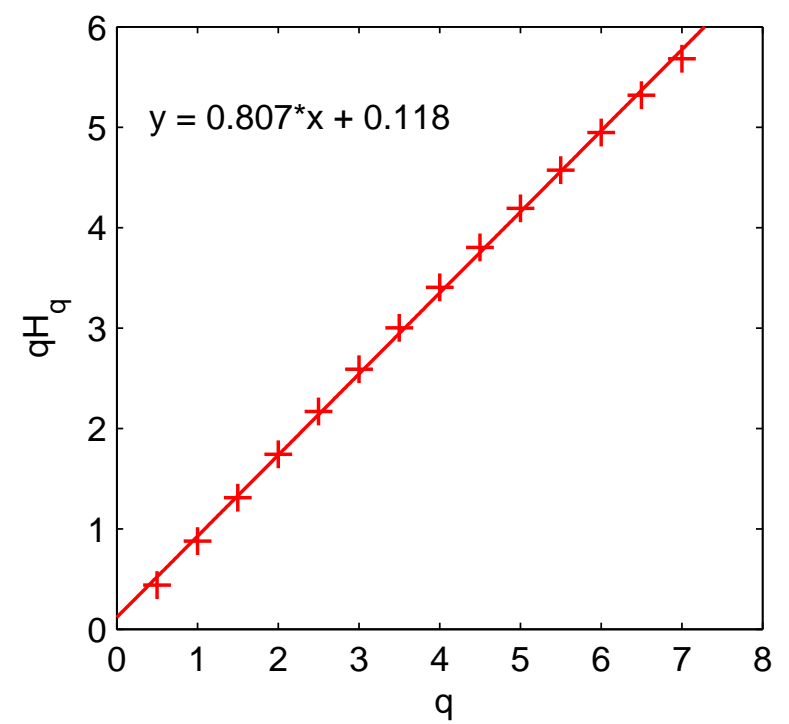

FIG. 10: (Color online) Variation of the product $q H_{q}$ with $q$ for the case with temperature gradient

$$
R / L_{T}=6.9
$$

no stationary part was detected in the time series. As already mentioned, we interpolate the computed data using cubic splines, in order to create data that are equally spaced in time.

Since the $x$ grid is limited to 128 data-points, we also divided the time direction into sets of 128 steps, and consider $128 \times 128$ sized squares as images. A sample image is presented in Fig. 11. We set a threshold value for the heat flux, and each value of heat flux in the computational grid equal to or above the threshold value is kept, and the values lower than the threshold are set to zero. In this way we isolate events of relatively large magnitude, with the threshold a free parameter. For each image we calculate the fractal dimension, using the box-counting method (see e.g., 35), and repeat the procedure for each simulation. The threshold value for the box counting method used is the mean value of the averaged in the radial direction heat flux, and also 1.5 times this mean value.

Results of the calculated fractal dimension $D$ are given in Tables II and III for the two threshold values. We see that with increasing temperature gradient the mean fractal dimension of the evolution is almost constant in the simulations. For threshold value the mean value of the averaged heat flux, Table II, we can see that $D$ is in the range $D=1.76-1.84$. Results when increasing the threshold to 1.5 times the mean value are given in Table III and give a $D$ in the range $D=1.45-1.56$. The maximum values of the dimension are also almost constant, with values 1.9 and 1.7, respectively. The minimum values of the dimension are more diverse in values than the maximum values.

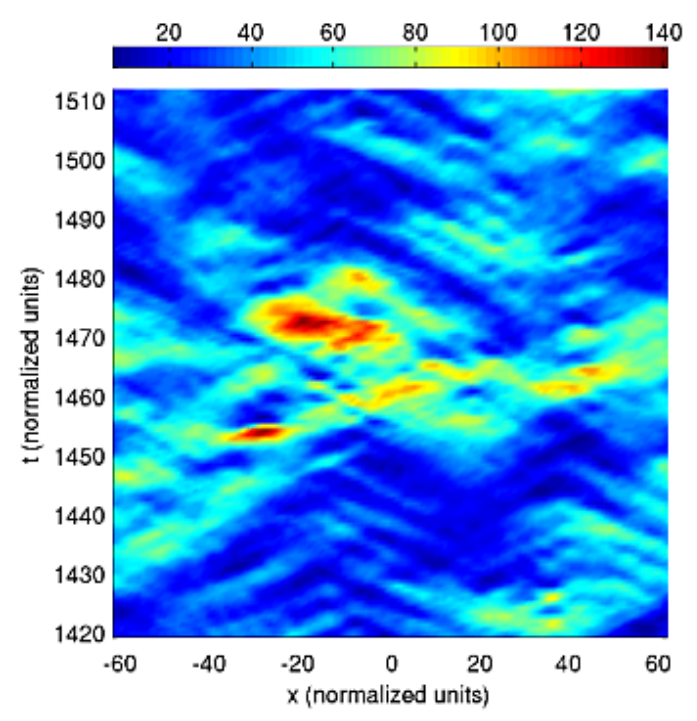

FIG. 11: Large events in a $128 \times 128$ sample image of heat flux in the radius time plane from a simulation with $R / L_{T}=6.5$.

TABLE II: Values of fractal dimension $D$ for a threshold value equal to the mean value of the radially averaged heat flux: mean value $D_{\text {mean }}$ of the $128 \times 128$ images, maximum value $D_{\max }$, minimum value $D_{\min }$.

\begin{tabular}{crrr}
\hline \hline Temperature Gradient & $\mathbf{D}_{\min }$ & $\mathbf{D}_{\max }$ & $\mathbf{D}_{\text {mean }}$ \\
\hline 3.5 & 1.677 & 1.911 & 1.84 \\
\hline 4.0 & 1.734 & 1.934 & 1.84 \\
\hline 4.5 & 1.426 & 1.95 & 1.76 \\
\hline 5.0 & 1.713 & 1.903 & 1.80 \\
\hline 6.0 & 1.487 & 1.939 & 1.79 \\
\hline 6.5 & 1.669 & 1.909 & 1.81 \\
\hline \hline
\end{tabular}

TABLE III: Values of fractal dimension $D$ for a threshold value equal to 1.5 times the mean value of the radially averaged heat flux: mean value $D_{\text {mean }}$ of the $128 \times 128$ images, maximum value $D_{\max }$, minimum value $D_{\min }$.

\begin{tabular}{crrr}
\hline \hline Temperature Gradient & $\mathbf{D}_{\min }$ & $\mathbf{D}_{\max }$ & $\mathbf{D}_{\text {mean }}$ \\
\hline 3.5 & 1.043 & 1.721 & 1.45 \\
\hline 4.0 & 1.213 & 1.781 & 1.54 \\
\hline 4.5 & 0.545 & 1.874 & 1.56 \\
\hline 5.0 & 1.276 & 1.689 & 1.53 \\
\hline 6.0 & 1.08 & 1.76 & 1.51 \\
\hline 6.5 & 1.095 & 1.714 & 1.47 \\
\hline \hline
\end{tabular}




\section{B. Cluster analysis}

For the detection of structures mainly in the radial direction, we perform an analysis that determines the structures (clusters) and counts their radial extent and number. We again set a threshold value for the heat flux and retain all values of the observable above this threshold, all other values are set to zero. In this way isolated radially extended structures are made identifiable. Each structure is considered as separate if there is at least an empty grid point surrounding it in every direction. We then determine the extent of these structures in radial direction and calculate the probability distribution functions of the radial extents.

We use as threshold values 1.5, 1.8, 1.9, 2.0 times the mean value of heat flux. A sample image of the structures is given in Fig. 12 for the simulation with $R / L_{T}=6.5$ and with threshold value 1.5 times the mean value of the heat flux. We see that the structures have a quite large radial extent within the flux tube, and thus they can be considered as candidates for avalanches, see below.

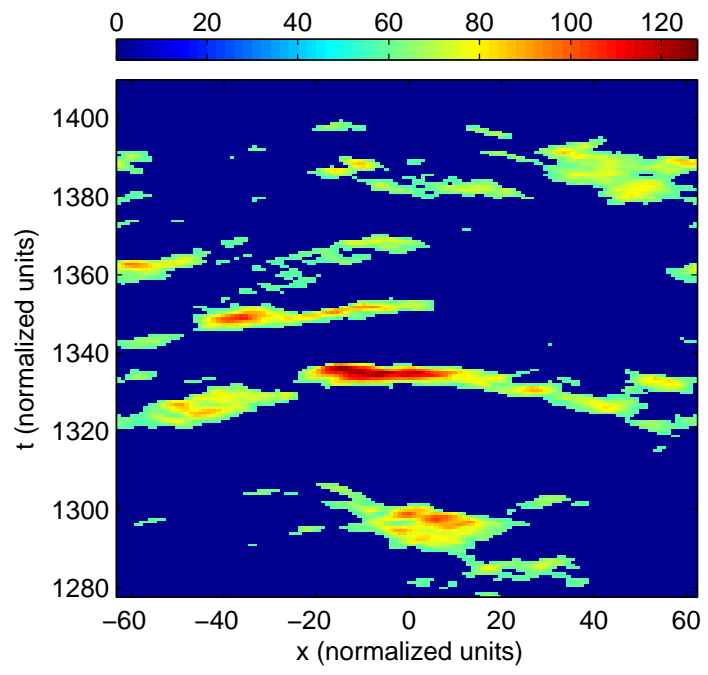

FIG. 12: (Color online) Radially extended structures in a contour plot of the heat flux with threshold 1.5 times

the mean value of heat flux, for simulations with

$$
R / L_{T}=6.5
$$

The histogram of the radial extent of a selected case is presented in Fig. 13. We can identify a clear power law shape, with two main regions, a region of low slope $\sim 2$ and bigger extent (region $\mathrm{A}$ ) and a region with high slope and smaller extent (region B). The overall extent of the histogram is limited by the relatively small size of the grid in the radial direction. We calculate the slope of the power laws with a fit, and the variation of the exponent of the power law with the temperature gradient in the first region is shown in Fig. 14. The variation is quite small, with almost constant values $1.6 \sim 1.7$ with exception the case $R / L_{T}=4$. This region concerns events of small to medium extent and has structures with power-law shapes whose indexes are also almost unaffected by the threshold set. The exponent of the power law index of the radially more extended structures in the second (B) region, has higher values and seems to be affected by the threshold. Yet the values can not much be interpreted due to the small extent of the power laws.

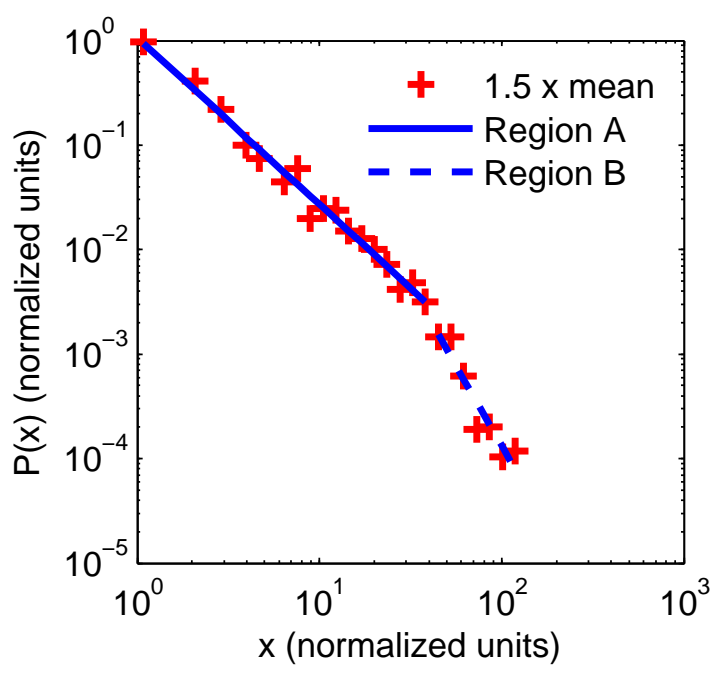

FIG. 13: Histogram of the radial extent of structures of heat flux with values above a threshold of 1.5 times the mean value and for the simulation with $R / L_{T}=6.5$. The solid line fits region $\mathrm{A}$, and the dashed line fits region $\mathrm{B}$.

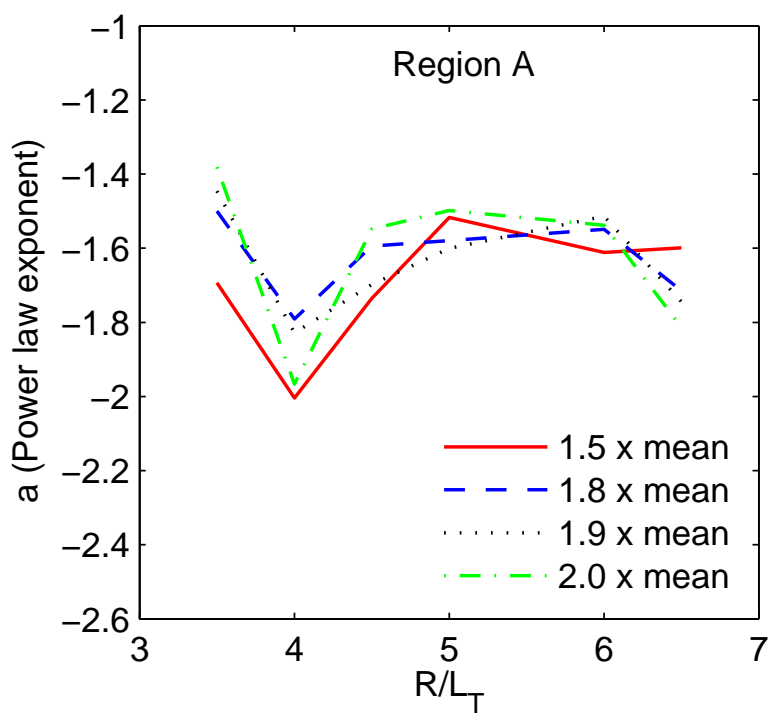

FIG. 14: (Color online) Variation of the power law index of the histograms of the radial extent of structures with the temperature gradient in region $\mathrm{A}$. The threshold is set to 1.5 (red solid line), 1.8 (blue dashed line), 1.9 (black dots), 2.0 (green dashed-dotted line), times the mean value of the radially averaged time series. 


\section{Two dimensional autocorrelation function}

The autocorrelation function is defined as the cross correlation of the signal and a lagged version of itself. In our case the signal consists of heat flux in the $x-t$ plane, and we calculate the two dimensional autocorrelation function.

For the calculation we use the method based on the Wiener - Khinchin theorem, which allows computing the autocorrelation from the data $q(x, t)$ with two Fast Fourier transforms (FFT). First, we subtract the mean value from the data. We then compute the autocorrelation function of the data by Fourier transforming them into the frequency domain, taking the modulus of the spectral coefficients, and then performing the inverse Fourier transform.

For each one of the simulations and for the purpose of Fourier transforms, a data grid of heat flux, that consists in 128 points in the radial $(x)$ direction, and 4096 points in the time $t$ direction was selected as the highest values which are both powers of two. Also we do not show the redundant negative time lags.

We calculate the velocity of possibly traveling structures appearing in the autocorrelation function. At every time lag, we locate the position of the maximum value of the autocorrelation function above a minimum value of 0.3 , for both positive and negative spatial lags $\delta x$. This produces lines in the time-lag radial-lag plane and the calculation of the slope of a linear fit to these lines, wherever this is possible, gives the velocity of structures in units of the thermal speed.

At low temperature gradients $R / L_{T}=3.5,4,4.5$ we observe that there are no traveling structures in the radial direction, see the example in Fig. 15. As the temperature gradient increases we find that for the simulations with $R / L_{T}=6.0$ and 6.5 there are radially traveling structures. We make a linear fit for these two cases and calculate the velocity of these structures. In the case of the temperature gradient $R / L_{T}=6$ we find for positive lags, a velocity $v=2.79$, while for negative lags $v=-2.57$. In the case of the temperature gradient $R / L_{T}=6.5$ we find for positive lags, a velocity $v=2.96$, while for negative lags $v=-2.75$. In both cases, the values of the velocity at negative and positive spatial lags, are close, with the velocity of the positive spatial lags being larger. Also, there is an increase of these values with the increase of the temperature gradient.

Because in our analysis so far, we have seen that the radial structures have almost the same size distribution and the fractal dimension of the images in the radius-time plane is almost constant, the above result is interesting, because we find that these structures start traveling only at large temperature gradients. So although these structures exist even with smaller gradients their radial motion starts above a certain threshold in the temperature gradient.

In order to investigate this further we calculate according to Ref. 18 the tilting parameter $P=\operatorname{Im}[(d q / d x+$
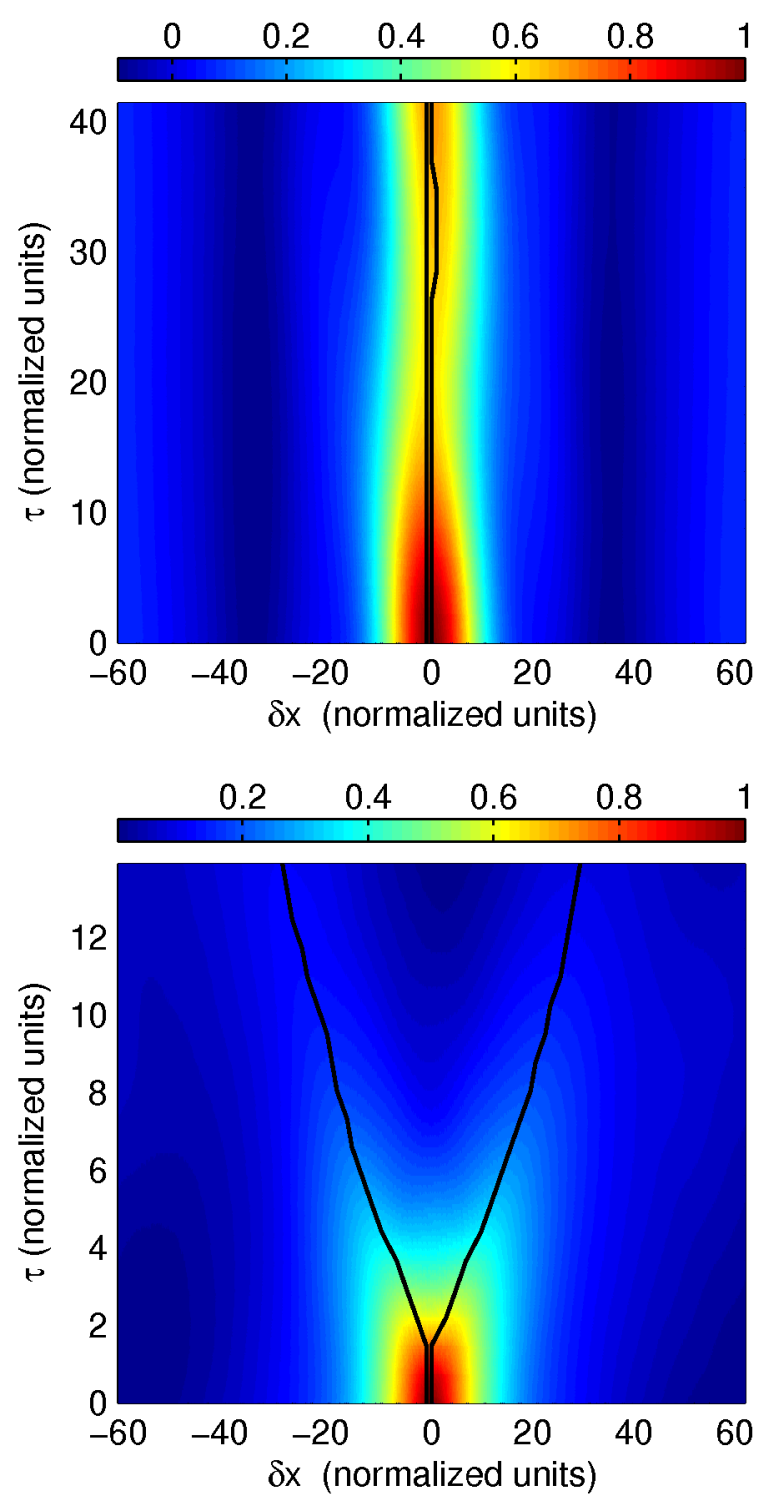

FIG. 15: (Color online) Autocorrelation function for the simulations with gradients $R / L_{T}=3.5$ (top) and $R / L_{T}=6.5$ (bottom). The black lines show the local maximum value of the autocorrelation function.

$\left.\imath d q / d t)^{2}\right]$ where $q$ is the heat flux. This quantity measures the dominant direction of diagonally aligned structures. Spatial locations with positive $P>0$ correspond to events propagating to smaller $x$ with increasing time and vice versa for $P<0$. The mean value of $P$ is almost zero for all simulations meaning that we have equally tilted structures in both directions. The standard deviation $\sigma$ of $P$ though is increasing with the temperature gradient, as can be seen in Fig. 16. The increase of the tilting of structures is related to the motion of structures, which we now can consider to be avalanches at the large temperature gradients. 


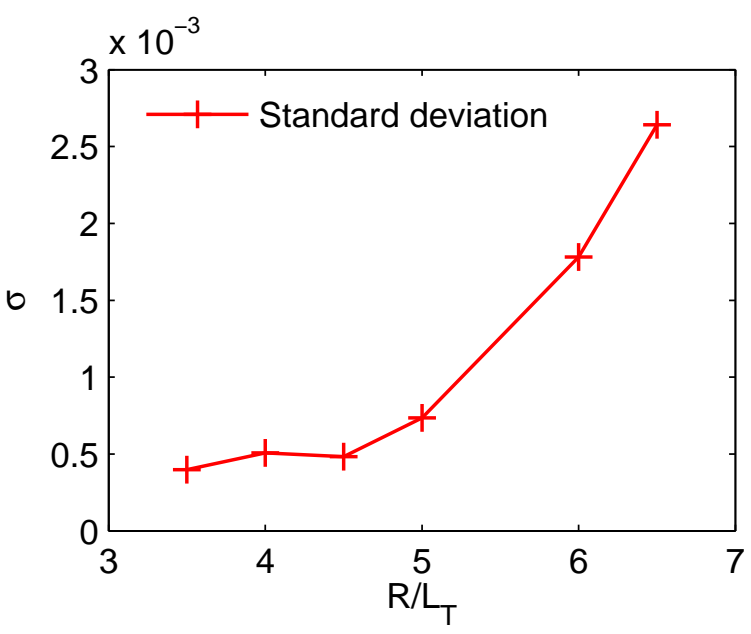

FIG. 16: (Color online) Variation of the standard deviation $\sigma$ of the tilting parameter $P$ with the temperature gradient.

\section{DISCUSSION}

Since Soc is considered to be linked to systems in a marginal stability state, we start the simulations from a low temperature gradient very close to the linear threshold, where the turbulence drive is low, and we then gradually increase the gradient. We could thus divide the simulations into three regimes: (a) a regime $R / L_{T} \sim 3.5-4.5$ close to the linear threshold for the fastest growing mode, where the heat flux transport is very low, (b) the Dimits shift $^{29}$ regime $R / L_{T} \sim 5-6$, near where the heat flux transport still remains relatively small, and (c) the stiff regime $R / L_{T} \geq 6.0$, where the heat flux strongly depends on and increases as the temperature gradient increases.

In the first regime, close to the linear threshold, the fluctuation level of the various observables is low, mainly of the density. Zonal flows appear, which give rise to a finite shearing rate. In Fourier space, we observe that the amplitude of modes non-linearly interacting is significant for only a limited number of them, for the observables of density and temperature in the direction perpendicular to the magnetic field. For the other observables, e.g. the electrostatic potential, the modes corresponding to zonal flows have the largest amplitude. In the time-radius plane, we observe heat flux structures with a quasi-oscillating behavior, rarely overlapping between them, and when so, giving rise to larger structures. When examining the contribution of each $k_{y}$ mode to the total heat flux, we can see that only a small number of modes have a significant contribution. These modes are typically localized next to the largest linear growth rate, while most of the other modes included in the simulation have a linear growth rate close or equal to zero.
This oscillating behavior of heat flux structures could be understood as a consequence of the limited number of modes that are significant in the non-linear interactions and participate in the transfer of energy. We could say that heat flux structures have a streamer-like behavior. This behavior can also be considered responsible for the value of the Hurst exponent to be close to unity, since the quasi-oscillating heat flux structures are highly correlated. Also, it should lead to peaks appearing in the power-spectrum at specific frequencies, which is observed in some of the cases simulated (e.g. Fig. 4 for the case with $R / L_{T}=3.5$ ), but radially averaging the time series of the heat flux, as we did it in our analysis, tends to smear out clear peak-shaped structures.

The Dimits shift is basically the nonlinear upshift of the threshold for collisionless, electrostatic ITG turbulence, which is caused by undamped, self-generated zonal flows. In the regime of the Dimits shift, the number of unstable and non-linearly interacting modes with significant amplitude of the density and the temperature in the direction perpendicular to to magnetic field increases both in $k_{x}$ as well as in $k_{y}$ space. The amplitude of the density fluctuations remains at low values though, while the amplitude of the temperature fluctuations increases significantly. Heat flux structures gradually increase their radial extent, and also their overlapping is increasing but still limited, and there is no significant degree of radial traveling structures. A gradual increase in their tilting is also observed, and also the shearing rate produced by zonal flows increases. The oscillating behavior of heat flux structures, although present, diminishes, while larger magnitude heat flux structures appear. These heat flux structures could be described to have a streamer-like/bursty behavior. This behavior, as already stated, makes the Hurst exponent in the mesoscale region to remain close to unity. Turbulence is, relatively to the previous regime, increased and more developed. Also, the timeseries of the averaged heat flux appear to be more intermittent in this regime. This could explain the increase of the power law exponent with the temperature gradient, yet there is no self-organization. The Dimits shift regime is one where free energy is transferred to the zonal flows, with relatively little remaining in the drift waves. Thus this regime is dominated by zonal flows.

In the stiff regime with large temperature gradients $R / L_{T} \geq 6.0$, the level of fluctuations of the electrostatic potential increase, and the number of modes of the electrostatic potential in Fourier space that are interacting non-linearly and have significant amplitude also increases. The latter also stands for the parallel velocity, while for the density and the temperature almost all modes taken into account by the simulation set-up interact non-linearly with large amplitudes in Fourier space. Strong zonal flows shear turbulent eddies for all the mentioned observables. In the radius-time plane, radially traveling heat flux structures appear and overlap. As described in our analysis, their attributes (tilting, radial traveling, large tail in the two dimensional autocorrela- 
tion function) allows us to characterize such structures as avalanches. So the non linear interaction between zonal flows and drift waves seems to lead to self-organization, as the values of the power spectrum indices, the Hurst exponent in the mesoscale region, as well as other findings, tend to resemble those produced by direct Soc models. Also, it has been reported in many cases ${ }^{31}$ that this turbulence regime should be considered as a self-regulating, two-component system consisting of the usual drift wave spectrum and zonal flows.

An interesting point to comment on is the local minimum in the power-law exponent (Figure 5). This can be interpreted based on the work of Ref. 32 and the above analysis. At lower temperature gradients, large non-overlapping events with significant radial extent appear and are of quasi periodic nature, having as already mentioned a streamer-like behavior which results in an exponent in the intermediate frequency region between $1 \sim 2$. As turbulence begins to develop with the increase of the temperature gradient to intermediate values, this quasi periodic nature diminishes. Independent, yet still with a tendency not to over-lap, large scale heat flux events with no radial traveling and with a streamerlike/bursty behavior lead then to a regime where the exponent shows its minimum. Further increase of the temperature gradient increases the number of the large heat flux events, which become tilted and overlap having a more avalanche-like behavior. The latter gradually increases the value of the exponent, which finally, when the gradient exceeds $R / L_{T} \geq 6.0$, leads to $1 / f$ noise.

The skewness and kurtosis show a minimum in the same range of temperature gradients $(4-5)$ as the powerlaw exponents. Thus, it seems that the two statistics follow each other: there is a reduction in the temporal correlations in this region of gradients that is accompanied by an increased 'Gaussianity' of the heat-flux histograms. We can conclude that we see a transition from a quasi-periodic, well correlated, slightly non-Gaussian regime at the lowest gradients, to turbulent, correlated and self-organized, again slightly non-Gaussian regime, at the highest gradients. The regime of intermediate gradients is the transient regime, where the developing turbulence can be described as not yet much self-organized and correlated noise with close to Gaussian statistics.

The main goal of this work is to conclude whether ITG mode driven gyrokinetic turbulence reaches a state of self-organization. This is quite a difficult task since the definition of a Soc state is still a field of debate. In brief, one can distinguish three definitions of Soc, a phenomenological ${ }^{3}$ definition, an operational ${ }^{10}$ definition, and a theoretical ${ }^{3}$ definition. The theoretical definition (driven, spatially extended, non-linear systems that are in a minimally (marginally) stable state) is vague in the sense that it expresses only a necessary prerequisite for Soc to appear. The operational definition can be understood as set of guiding rules for the construction of explicit Soc systems (e.g. the system is spatially extended and driven locally, there are local threshold dependent instabilities, and there is a local relaxation mechanism that stabilizes the instabilities). Again, the operational definition enumerates basically necessary conditions for the appearance of Soc, and in any case, using either the theoretical or the operational definition, the 'proof' that a Soc state is reached can be made only by using the phenomenological definition of Soc, which describes a basic set of statistical properties of the Soc state (the appearance of avalanches, with power-law shaped distribution functions and power-spectra, self-similarity and long range correlations etc.).

In the frame of these considerations, we apply the phenomenological definition of Soc in our work. Thus, according to the phenomenological definition, some important characteristics of a Soc state include a characteristic form of the power spectrum having three distinct regions (Ref. 32, and see also the respective comment therein): A high frequency region where the exponent is approximately -4 , the middle to low frequencies region (the avalanche overlapping region) where the power law exponent is approximately -1 and the region of low frequencies where the spectrum is flat and has an exponent approximately 0 . Although in our simulations, the power spectrum has a definite multiple power law form, the exponents are closer to the usual values of a Soc state as the temperature gradient $R / L_{T} \geq 6.0$ in the simulations. Since the simulation with gradient $R / L_{T}=5.5$ had no stationary part for the analysis, a precise determination of the gradient where the usual power spectrum shape of a Soc system appears cannot be made. The latter gradient value concerns of course only the simulations performed in this work.

Long range correlations are another characteristic of a system in a Soc state. A usual method for their detection is the calculation of the Hurst exponent, which in this work is performed with two different methods, the $R / S$ analysis and the structure function method, and which give similar results. A usual value of the Hurst exponent for a system in a Soc state is $H \sim 0.7-0.8$ (e.g. Ref. 9). In our case these values appear also, as can be seen in Figures 7 and 9, for the same large values of the temperature gradient $R / L_{T} \geq 6$.

The previous point is also related to a third phenomenological criterion, the existence of an algebraic tail in the autocorrelation function. This can be detected in the calculation of the one or two dimensional autocorrelation function, which falls off quite rapidly for the simulations with low temperature gradient, while for those simulations preformed for gradients $R / L_{T} \geq 6.0$ the autocorrelation function stays above the $1 / e$-limit for larger values of the time lag (see e.g. Fig. 15).

Finally, as mentioned in Ref. 4, in a Soc state, coherent avalanches can be detected to grow and shrink, some are seen to propagate 'up-hill' while others are seen propagating 'down-hill', and this dual propagation represents a characteristic feature of the Soc state. In our study, we observe propagation of avalanches for the already mentioned high temperature gradient values (see 
e.g. Figures 15, 16).

We must mention though that in local simulations the mean temperature gradient stays constant during the simulations, so that, applying the theoretical definition of Soc, and studying the evolution of the temperature profile and whether it reaches a subcritical state, which also is a characteristic of Soc, is not feasible in the presented simulations. Having the latter in mind, we conclude from the above analysis that a Soc state is probably reached for the simulations that have $R / L_{T} \geq 6.0$, or, more carefully formulated, our findings are largely compatible with the system being in the state of Soc for large gradients. A careful formulation also is appropriate since one basic phenomenological property of Soc is absent, namely power-law shaped distribution functions of system variables, such as of the heat flux in our case.

\section{CONCLUSIONS}

We have made local non linear gyrokinetic simulations with varying temperature gradients, starting just above the linear critical threshold and extending up to the standard value of the CBC scenario. We made a statistical analysis of the time series of the averaged heat flux and of the heat flux as a function of radius and time, applying various statistical tools.

In the analysis of the radially averaged heat flux we find some of the characteristics of Soc systems, mainly as the temperature gradient increases. At low gradients only a few modes non-linearly interact with a significant amplitude and turbulence is not fully developed. When all modes interact non linearly with almost the same amplitude, we observe the multiple power law form of the power spectra, as well as the $1 / f$ dependency of the power spectrum in the intermediate frequency region. The Hurst exponent $H \sim 0.8$ is estimated with the $R / S$ method and also by using structure functions. This value shows the existence of long range correlations, a main ingredient of Soc systems. Histograms of heat flux do not have a power law form, which would be expected in a system exhibiting Soc. Instead, they have an almost Gaussian shape with only a small tail and with positive skewness and kurtosis.

In the radius - time plane we find that, although the topology of the heat flux patterns does not change significantly, when characterized by the fractal dimension or the histograms of the radial extent of the structures, which both show only slight variations, the motion of structures in the radial directions appears only at the largest gradient values. Also, the increasing tilting of these structures as the temperature gradient increases is closely related to their radial motion. We thus conclude that there are radially extended structures for all temperature gradients, only for large gradients though they are traveling and can be called avalanches.

These results, obtained with a gyrokinetic code, resemble experimental observations, although a direct compar- ison can be made only with care since we have restricted our study to local non linear simulations. Experimental measurements made at the plasma edge of Tokamaks show the multiple power law form, as well as a close to $1 / f$ dependency in the intermediate frequency region of the power spectra (e.g., see Refs. 11-17), which was also found in this study for large temperature gradients. The index in the high frequency region though is usually slightly smaller than the one found in this work. The Hurst exponent calculated from experimental data with the use of the same methods as done here $(\mathrm{R} / \mathrm{S}$ analysis, structure function), show for the mesoscale region values ranging usually between $0.7 \sim 0.8$ (e.g., see Refs. 11-13, and 16), similar to our findings.

By applying a phenomenological definition of a SoC state, we can conclude that a Soc state is probably reached for the simulations that have $R / L_{T} \geq 6.0$, with one characteristic Soc feature being absent, namely power-law shaped distribution functions. Finally, the fact that some characteristics of self organized criticality can be reproduced by a gyrokinetic code, shows that these can arise from the physical content of the gyrokinetic equations and mainly the non linearities. In the future, an extension of this kind of work will be made, including global, flux and gradient driven simulations, for a direct comparison with the experimental data, as well as with the findings of the local simulations presented in this study for large temperature gradients.

\section{ACKNOWLEDGMENTS}

This work is supported by (a) the National Programme for the Controlled Thermonuclear Fusion, Hellenic Republic, (b) the European Union's Horizon 2020 research and innovation programme under grant agreement number 633053 (M.M., H.I., L.V.). The authors gratefully acknowledge the computing time granted on the supercomputer JUROPA/HPC-FF at Jülich Supercomputing Center (JSC). The views and opinions expressed herein do not necessarily reflect those of the European Commission.

\section{Appendix A: Statistical Tools}

The theoretical framework of the statistical tools used throughout this work is given below.

\section{Hurst exponent}

In the $R / S$ analysis a self-similar scaling

$$
\lim _{\tau \rightarrow \infty} \frac{R}{S}(\tau)=\lambda \tau^{H}
$$

is sought, where $R$ and $S$ denote the range and the standard deviation, respectively, of the cumulative (inte- 
grated) time series $W_{k}, \tau$ denotes a time lag, $\lambda$ a constant and $H$ the Hurst exponent. For a time series segment of length $n$ which is a subset of the total data record of length $N(n \leq N), X \equiv\left\{X_{n}: n=1,2,3,4, \ldots, n\right\}$, corresponding to a stationary process, $W_{k}$ is given by $W_{k}=X_{1}+X_{2}+\cdots+X_{k}-k \bar{X}$, where $\bar{X}$ is the mean of $X$. The $R / S$ ratio is the ratio of the maximal range of the integrated signal normalized to its standard deviation. It is defined for a given time lag $n$ as

$$
\frac{R(n)}{S(n)}=\frac{\max \left(W_{1}, W_{2}, \ldots W_{n}\right)-\min \left(W_{1}, W_{2}, \ldots W_{n}\right)}{\sqrt{S^{2}(n)}}
$$

We divide the original data set into subsets according to the lag used, calculate for each subset the $R / S$ ratio and then calculate the mean value of all the subsets. A possible scaling of $R / S$ in the form of Eq. A1 is then searched for, which allows to determine $H$.

When $1>H>0.5$ there are long-range time correlations (persistence), for $0.5>H>0$ the time series has long-range anti-correlations (anti-persistence), if $H=1.0$ the process is deterministic and finally for time series with no correlations (i.e. purely random) $H=0$, and for Brownian motion $H=0.5$. The Hurst exponent is theoretically related to the fractal dimension D (see e.g., Ref. 35) as

$$
D=2-H
$$

\section{Structure function analysis - generalized Hurst exponent}

Assuming as before a stationary time series of length $N, X \equiv\left\{X_{n}: n=1,2,3,4, \ldots, N\right\}$, we can create again the cumulative series $W_{k}$, as already described in the previous section. For such a time series of length $N$, written now as $W\left(t_{i}\right)(i=1,2,3, \ldots N)$ the structure function of order $q$ is defined as:

$$
S_{q}=\left\langle\left|W\left(t_{i}+\tau\right)-W\left(t_{i}\right)\right|^{q}\right\rangle, q>0,
$$

where $\langle\cdot\rangle$ denotes the ensemble average. If the process is scale invariant and self-similar over some range of time lags $\tau_{1} \leq \tau \leq \tau_{2}$ then the $q$ th - order structure function is expected to scale as:

$$
S_{q}(\tau)=C_{q} \tau^{\zeta(q)}=C_{q} \tau^{q H(q)}
$$

where $C_{q}$ can be a function of $\tau$ which varies more slowly than any power of $\tau, \zeta(q)$ is the exponent of the structure function, and $H(q)$ is the Hurst exponent or self - similarity exponent of order $q$. Calculation of the Hurst exponent $H(q)$ allows the straight-forward identification of persistence or long time correlation, as in the $R / S$ analysis, as well as to determine the possibly monofractal or multifractal nature of the data. The structure function analysis should coincide with the $R / S$ analysis for the structure function order $q=2$.

\section{Power spectrum}

Also the power-spectrum can be used for the detection of self-similarity. The standard definition of selfsimilarity is that a process $X=\{X(t), t \geq 0\}$ is selfsimilar with self-similarity or Hurst, exponent $0<H<1$ if it satisfies

$$
X(\lambda t) \stackrel{d}{=} \lambda^{H} X(t)
$$

where $\stackrel{d}{=}$ means equality in distribution for any $\lambda>0$. Also, the stationary process $X$ is self-similar with selfsimilarity exponent $H$ if there exists a real number $\alpha \epsilon(0,2)$ such that

$$
\lim _{\tau \rightarrow \infty} \rho(\tau)=c_{\rho} \tau^{-\alpha}, 0<\alpha=2-2 H<2
$$

where $\rho(\tau)$ is the normalized autocorrelation function. In this case then the power spectral density exhibits the power law scaling

$$
\lim _{f \rightarrow \infty} S(f)=c_{S} f^{-\beta},-1<\beta=2 H-1<1
$$

with $c_{S}$ a constant, $f$ the frequency, $\beta$ the absolute value of the power spectrum index (see e.g., Ref. 35).

\section{Skewness and kurtosis}

Skewness is a measure of symmetry of a distribution, and kurtosis is a measure of whether the data are peaked or flat relative to a normal distribution. For data $X_{1}, X_{2}, \ldots, X_{N}$, the skewness $g_{1}$ is given as

$$
g_{1}=\frac{\frac{1}{N} \sum_{i=1}^{N}\left(X_{i}-\bar{X}\right)^{3}}{\left(\frac{1}{N} \sum_{i=1}^{N}\left(X_{i}-\bar{X}\right)^{2}\right)^{3 / 2}}
$$

where $\bar{X}$ is the mean value and $N$ is the number of data points. The skewness for a normal distribution is zero, as for any symmetric distribution. Negative values of the skewness indicate data that are skewed left and positive values indicate data that are skewed right.

The kurtosis is given as:

$$
\text { kurtosis }=\frac{\frac{1}{N} \sum_{i=1}^{N}\left(X_{i}-\bar{X}\right)^{4}}{\left(\frac{1}{N} \sum_{i=1}^{N}\left(X_{i}-\bar{X}\right)^{2}\right)^{2}}
$$

The kurtosis for a standard normal distribution is 3 and for this reason, we use the following definition of kurtosis (often referred to as "excess kurtosis")

$$
g_{2}=\frac{\frac{1}{N} \sum_{i=1}^{N}\left(X_{i}-\bar{X}\right)^{4}}{\left(\frac{1}{N} \sum_{i=1}^{N}\left(X_{i}-\bar{X}\right)^{2}\right)^{2}}-3
$$

In this way, the standard normal distribution has a kurtosis of zero, positive kurtosis indicates a "peaked" distribution and negative kurtosis indicates a "flat" distribution. 
${ }^{1}$ B. B. Kadomtsev, "Plasma turbulence" (Academic, London 1965).

${ }^{2} \mathrm{P} . \mathrm{H}$. Diamond and T. S. Hahm, "On the dynamics of turbulent transport near marginal stability", Phys. Plasmas 2, 3640 (1995).

${ }^{3}$ Bak P, Tang C, Wiesenfeld K, "Self-organized criticality: an explanation of $1 / f$ noise", Physical Review Letters 59 (4): 381384 (1987).

${ }^{4}$ Newman D. E, Carreras B. A, Diamond P. H, Hahm T. S, “ The dynamics of marginality and self-organized criticality as a paradigm for turbulent transport", Phys. Plasmas 3, 1858 (1996).

${ }^{5} \mathrm{X}$. Garbet and R. E. Waltz, "Heat flux driven ion turbulence Phys. Plasmas 5, 2836 (1998)

${ }^{6}$ X. Garbet, Y. Idomura, L. Villard and T.H. Watanabe, "Gyrokinetic simulations of turbulent transport ", Nucl. Fusion 50, 043002 (2010)

${ }^{7}$ Carreras B. A, Newman D, Lynch E.V, Diamond P.H, "A model realization of self - organized criticality for plasma confinement", Phys. Plasmas 3 (8) 1996.

${ }^{8}$ Garcia L, Carreras B. A, "Avalanche properties in a transport model based on critical-gradient fluctuation dynamics", Phys. Plasmas 12, 092305 (2005).

${ }^{9}$ R. Sánchez, D. E. Newman, B. A. Carreras, "Mixed SOC diffusive dynamics as a paradigm for transport in fusion devices", Nuclear Fusion, Vol. 41, No. 3 (2001).

${ }^{10}$ Isliker H, Pisokas Th, Strintzi D, Vlahos L, "A self-organized criticality model for ion temperature gradient mode driven turbulence in confined plasma", Phys. Plasmas 17, 082303 (2010).

${ }^{11}$ Gilmore M, Yu X. C, Rhodes T. L, Peebles W. A, "Investigation of rescaled range analysis, the Hurst exponent, and longtime correlations in plasma turbulence", Phys. Plasmas 9, 1312 (2002).

${ }^{12}$ Yu C. X, Gilmore M, Peebles W. A, Rhodes T. L, "Structure function analysis of long-range correlations in plasma turbulence", Phys. Plasmas 10, 2772 (2003).

${ }^{13}$ Politzer P. A, "Observation of Avalanchelike Phenomena in a Magnetically Confined Plasma", Physical Review Letters vol. 84 , number 6 (2000).

${ }^{14}$ Neto Rodrigues C, Guimarães - Filho Z. O, Caldas I. L, Nascimento I. C, Kuznetsov K. Yu, "Multifractality in plasma edge electrostatic turbulence", Phys. Plasmas 15, 082311 (2008).

${ }^{15}$ Scipioni A, Rischette, Bonhomme G, Devynck P, "Characterization of self-similarity properties of turbulence in magnetized plasmas", Phys. Plasmas 15, 112303 (2008).

${ }^{16}$ Y. H. Xu, S. Jachmich, R. R. Weynants, A. Huber, B. Unterberg, U. Samm, "Investigation of self-organized criticality behavior of edge plasma transport in Torus experiment of technology oriented research ", Phys. Plasmas 11, 5413 (2004).

${ }^{17} \mathrm{Yu}$ Y, Lu R, Wang Z, Wen Y, Yu C, Wan S, Liu W, “Statistical properties of turbulence in a toroidal magnetized ECR plasma", Physics Letters A, 372, (2008), 1081-1087.

${ }^{18}$ B. F. McMillan, S. Jolliet, T. M. Tran, L. Villard, A.Bottino et al., "Avalanchelike bursts in global gyrokinetic simulations", Phys. Plasmas 16, 022310 (2009).

${ }^{19}$ Jenko F, Dorland W, Kotschenreuther M and Rogers, "Electron gradient driven turbulence", Phys. Plasmas 7 1904-1910 (2000).

${ }^{20}$ Dannert T and Jenko F, "Gyrokinetic simulation of collisionless trapped - electron mode turbulence", Phys. Plasmas 12072309 (2005).

${ }^{21}$ Merz F, "Gyrokinetic Simulation of Multimode Plasma Turbulence", Ph.D. thesis (2008).

${ }^{22}$ T. Görler, X. Lapillonne, S. Brunner, T. Dannert, F. Jenko, F. Mertz, D. Told, "The global version of the gyrokinetic turbulence code GENE ", Journal of Computational Physics 230, 7053-7071 (2011).

${ }^{23}$ T. Görler, X. Lapillonne, S. Brunner, T. Dannert and F. Jenko, “ Flux and gradient driven global gyrokinetic simulations of tokamak turbulence", Physics of Plasmas 18, 056103 (2011).

${ }^{24}$ T. Görler, X. Lapillonne, S. Brunner, J. Chowdhury, T. Dannert, F. Jenko, B. F. McMillan, F. Merz, D. Told, "Non local effects in gyrokinetic turbulence simulations using GENE", Journal of Physics: Conference Series 260, 012011 (2010).

${ }^{25}$ J. Candy and R. E. Waltz, "Anomalous Transport Scaling in the DIII-D Tokamak Matched by Supercomputer Simulation", Phys. Rev. Lett. 91, 045001 (2003)

${ }^{26}$ G. Dif-Pradalier, P. H. Diamond, V. Grandgirard, Y. Sarazin, J. Abiteboul, X. Garbet, Ph. Ghendrih, A. Strugarek, S. Ku, and C. S. Chang, "On the validity of the local diffusive paradigm in turbulent plasma transport", Phys. Rev. E, 82:025401(R), 2010

${ }^{27}$ G. Dif-Pradalier, S. Ku, P.H. Diamond, C.S. Chang,Y. Sarazin, V. Grandgirard,J. Abiteboul, S. Allfrey, X. Garbet, Ph. Ghendrih, G. Latu, and A. Strugarek "Nonlocal dynamics of Turbulence, Transport and Zonal Flows in Tokamak Plasmas", Proc. 23rd IAEA Fusion Energy Conference Daejeon, 11-16 October 2010

${ }^{28} \ddot{O}$. D. Gürcan et al., "Structure of nonlocality of plasma turbulence", Nucl. Fusion 53, 073029 (2013)

${ }^{29}$ A. M. Dimits, A. M. Dimits, G. Bateman, M. A. Beer, B. I. Cohen, W. Dorland, G. W. Hammett, C. Kim, J. E. Kinsey, M. Kotschenreuther, A. H. Kritz, L. L. Lao, J. Mandrekas, W. M. Nevins, S. E. Parker, A. J. Redd, D. E. Shumaker, R. Sydora, J. Weiland, "Comparisons and physics basis of tokamak transport models and turbulence simulations " Phys. Plasmas 7, 969 (2000).

${ }^{30}$ X. Lapillonne, S. Brunner, T. Dannert, S. Jolliet, A. Marinoni, L. Villard, T. Görler, F. Jenko, and F. Merz, "Clarifications to the limitations of the $s$ - $\alpha$ equilibrium model for gyrokinetic computations of turbulence", Phys. Plasmas 16, 032308 (2009).

${ }^{31}$ P.H. Diamond, S. Champeaux, M. Malkov, A. Das, I. Gruzinov, M.N. Rosenbluth, C. Holland, B. Wecht, A.I. Smolyakov, F.L. Hinton, Z. Lin and T.S. Hahm, "Secondary instability in drift wave turbulence as a mechanism for zonal flow and avalanche formation", Nucl. Fusion 41, 1067 (2001).

${ }^{32}$ T.Hwa and M. Kardar, "Avalanches, hydrodynamics, and discharge events in models of sandpiles", Phys. Rev. A 45, 7002 (1992).

${ }^{33}$ H.E. Hurst, "Long-term storage capacity of reservoirs", Trans. Am. Soc. Civ. Eng. 116, 770 (1951).

${ }^{34}$ A. Davis, A. Marshak, W. Wiscombe and R. Cahalan, "Multifractal characterization of nonstationarity and intermittency in Geophysical fields : Observed, retrieved or simulated" J. Geophys. Res. 99 , 8055 (1994).

${ }^{35}$ Falconer, K., "Fractal Geometry", John Wiley \& Sons, Chichester (1990) 